\title{
Minimal surfaces and Reggeization in the AdS/CFT correspondence
}

\author{
R.A. Janik ${ }^{a, b}$ and R. Peschanski ${ }^{a}$ \\ ${ }^{a}$ Service de Physique Theorique CEA-Saclay \\ F-91191 Gif-sur-Yvette Cedex, France \\ ${ }^{b}$ M.Smoluchowski Institute of Physics, Jagellonian University \\ Reymonta 4, 30-059 Cracow, Poland
}

June 13, 2021

\begin{abstract}
We address the problem of computing scattering amplitudes related to the correlation function of two Wilson lines and/or loops elongated along light-cone directions in strongly coupled gauge theories. Using the AdS/CFT correspondence in the classical approximation, the amplitudes are shown to be related to minimal surfaces generalizing the helicoid in various $A d S_{5}$ backgrounds. Infra-red divergences appearing for Wilson lines can be factorized out or can be cured by considering the IR finite case of correlation functions of two Wilson loops. In non-conformal cases related to confining theories, reggeized amplitudes with linear trajectories and unit intercept are obtained and shown to come from the approximately flat metrics near the horizon, which sets the scale for the Regge slope. In the conformal case the absence of confinement leads to a different solution. A transition between both regimes appears, in a confining theory, when varying impact parameter.
\end{abstract}




\section{Introduction}

The theoretical calculation from "first principles" of high energy scattering amplitudes in the so-called "soft" regime of QCD is among the oldest and yet unsolved problem of strong interaction physics. The main reason is that it requires a good understanding of 4-dimensional gauge field theories at strong coupling which we do not possess till now. In view of the recent developments of the AdS/CFT correspondence [1, 2] it is thus natural to address this problem in the new setting proposed in this way. An exact correspondence for QCD is not yet known, however useful information can be obtained from known realizations for confining theories.

We would like to discuss relevant physical properties of scattering amplitudes at high energy expected from the S-Matrix theory of strong interactions [3]. In particular, Reggeization of scattering amplitudes is expected to occur, i.e. high-energy two-body amplitudes behaving as $A(s, t)=s^{\alpha(t)} \times$ (prefactors), where $s, t$ are the well-known Mandelstam variables. $\alpha(t)$ is the Regge trajectory corresponding to singularities of partial waves at $j=\alpha(t)$ in the t-channel. Unitarity, analyticity and crossing relations implied by the S-Matrix theory impose constraints on $\alpha(t)$. In particular the Froissart bound [4] implies that $\alpha(t=0) \leq 1$ and the prefactors of the amplitude are at most like $\log ^{2} s$. Note that the Froissart bound assumes an underlying confining field theory, or at least a mass gap, since the scale of the bound is fixed by the particle of smallest mass (e.g. the pion).

In [5], we considered large impact parameter and high energy scattering of colourless states for $S U(N)$ supersymmetric gauge theories in the strong coupling, large $N$ limit using the AdS/CFT correspondence. The gauge theory scattering amplitude is linked with a correlation function of tilted Wilson loops elongated along the light-cone directions [6, 7, 8, 9]. In the AdS/CFT correspondence, these correlation functions are related to minimal surfaces in the $A d S_{5}$ geometry which have the Wilson loops as boundaries. The case considered in our previous paper Ref. [5] involved disjoint minimal surfaces and thus the necessity of including supergravity field exchanges between the two corresponding string worldsheets. The dominant contributions were identified and all correspond to real phase shifts, i.e. purely elastic scattering. In particular, the contribution of the bulk graviton gives an unexpected "gravity-like" $s^{1}$ behaviour of the gauge theory phase shift in a specific range of energies and (very) large impact parameters. 
The main but stringent difficulty which limited the scope of Ref. [5] was that the weak field approximation in supergravity was shown to be broken unless the impact parameter $L$ was sufficiently large, namely $\frac{L}{a} \gg s^{2 / 7}$, where $a$ is the transverse extension of the Wilson loop. If the above condition is not met, the produced gravitational field in the dual AdS theory becomes strong, preventing perturbative calculations to be done in this background.

We will concentrate on a situation where the difficulty with supergravity field exchanges does not arise, since there exists a single connected minimal surface which gives the dominant contribution to the scattering amplitude in the strong coupling regime, i.e. when $\alpha^{\prime} \rightarrow 0$. This will allow us to extend our study to small impact parameters, where inelastic channels are expected to play an important rôle.

In this approach we will start by considering the correlation function of two Wilson lines elongated along the two light-cone directions, a configuration which can be used for the description of highenergy quark-quark or quark-antiquark amplitudes in gauge theories [6, 7, 8]. The rôle of the quarks in the AdS/CFT correspondence will be played, as in [10], by the massive $W$ bosons arising from breaking $U(N+1) \rightarrow U(N) \times U(1)$. The case of IR finite correlators of Wilson loops will be dealt with in a second stage.

The plan of our paper is as follows: in section 2, we will analyze the correlation function of Wilson lines leading to an evaluation of $q \bar{q}$ and $q q$ scattering amplitudes at high energy. This will be done in the context of the black hole geometry in AdS space [11] (static Wilson loops were first studied in this background in [12, 13]), where one can use a flat metric as a good approximation scheme near the horizon. We analyze the factorizable structure of the IR divergences and isolate a cut-off independent inelastic amplitude leading to reggeization. In section 3, we consider the so-called "conformal" case of the AdS/CFT correspondence for $\mathcal{N}=4$ supersymmetric $S U(N)$ gauge theory, where the $A d S_{5}$ metric gives rise to a different minimal surface solution. The problem of the cancelation of the infra-red divergences is analyzed by considering Wilson loop correlators in section 4, leading to the (approximate) derivation of scattering amplitudes between colourless states, while the conclusions and open problems are pointed out in the final section. 


\section{Wilson lines and minimal surfaces in "quasi-flat" geometry}

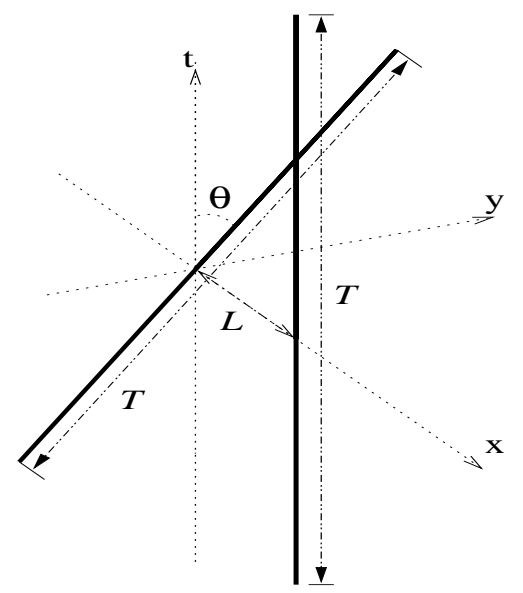

Figure 1: Geometry of the Wilson lines in euclidean space.

Let us start by defining an appropriate gauge theory observable for $q \bar{q}$ scattering amplitudes $A(s, t)$. It is convenient to pass from transverse momentum $t=-q^{2}$ to impact parameter space

$$
\frac{1}{s} A(s, t)=\frac{i}{2 \pi} \int d^{2} l e^{i q \cdot l} \tilde{A}(s, l)
$$

where $l$ is the 2-dimensional impact parameter (in the following we will denote its modulus by $L$ ), and $\tilde{A}$ is the amplitude in the impact parameter space.

In the eikonal approximation the impact parameter space amplitude for $q \bar{q}$ scattering is given by a correlation function of two Wilson lines [6, 8, 8] which follow the classical straight line quark trajectories $W_{1} \longrightarrow x_{1}^{\mu}=p_{1}^{\mu} \tau$ and $W_{2} \longrightarrow x_{2}^{\mu}=x_{\perp}^{\mu}+p_{2}^{\mu} \tau$, with $\left|x_{\perp}\right|=L$, see Fig.1. The IR cut-off will correspond to a fixed temporal extent of the lines $-T<\tau<+T$.

The AdS/CFT correspondence gives a recipe [10, 14] for calculating this correlation function through

$$
\left\langle W_{1} W_{2}\right\rangle \equiv \tilde{A}(s, l)=e^{-\frac{1}{2 \pi \alpha^{\prime}} A_{\text {minimal }}}
$$


where $\left\langle W_{1} W_{2}\right\rangle$ is the Wilson line correlator $\rrbracket, \alpha^{\prime}=1 / \sqrt{2 g_{Y M}^{2} N}$ in units of the AdS radius, and $A_{\text {minimal }}$ is the area of the minimal surface in the appropriate background geometry (e.g. $A d S_{5} \times S^{5}$ for the conformal $\mathcal{N}=4 \mathrm{SYM}$, an $A d S$ black hole [11, 12, 13] among other geometries [15] for confining theories) bounded by the Wilson line segments limited by the cut-off $T$. A different approach to discuss the minimal surface problem in the conformal $A d S_{5}$ was considered in [16], which concentrated on the elastic part of the amplitude.

Since the disjoint contour formed by the two Wilson line segments is not closed, the procedure for finding a minimal surface is ambiguous. We will adopt a prescription for finding the minimal surface for infinitely long lines and then truncating it to a finite temporal extent parameterized by the IR cutoff $T$. This implicitly consists of forming a "big" Wilson loop closed at large temporal distance by curves drawn on the infinite minimal surface.

In turn, this procedure defines the appropriate colour decomposition of the associated amplitude. Using the well known colour decomposition $t_{i j}^{a} t_{k l}^{a}=-1 / 2 N \delta_{i j} \delta_{k l}+1 / 2 \delta_{i l} \delta_{j k}$, we have

$$
\tilde{A}(s, l) \equiv N\left\{\tilde{A}_{0}(s, l)+\frac{1}{2} \tilde{A}_{N^{2}-1}(s, l)\right\}
$$

where $\tilde{A}_{0}$ (resp. $\tilde{A}_{N^{2}-1}$ ) are the amplitudes in the singlet (resp. adjoint) representations.

Using the same strategy as in our first paper [5], we will perform the calculation with euclidean signature for Wilson lines in the boundary $\mathbb{R}^{4}$ forming a relative angle $\theta$ in the longitudinal plane and then we will make an analytical continuation into Minkowski space by rotating the euclidean time coordinate clockwise and the angle anticlockwise (see [17] in this context):

$$
\begin{aligned}
\theta & \longrightarrow-i \chi \sim-i \log \frac{s}{m^{2}} \\
T & \longrightarrow i T .
\end{aligned}
$$

Note that a priori there is an ambiguity in making the analytical continuation depending on the precise choice of the path. This phenomenon did not appear in the context of large impact parameter near forward scattering discussed in [5] since there, the $\langle W W\rangle$ correlation

\footnotetext{
${ }^{1}$ The free propagation of the $q$ and $\bar{q}$ states is not included in the correlator $\left\langle W_{1} W_{2}\right\rangle$, which is thus implicitly normalized by $1 /\left\langle W_{1}\right\rangle\left\langle W_{2}\right\rangle$.
} 
function had only simple poles in the complex $\theta$ plane. In the case considered in this paper the analyticity structure contains branch cuts in the complex plane which have to be taken into account.

\section{$A d S$ black hole solution and its flat space approximation}

In 11] a proposal was made that a confining gauge theory is dual to string theory in an $A d S$ black hole (BH) background the relevant part of which can be written as

$$
d s_{B H}^{2}=\frac{16}{9} \frac{1}{f(z)} \frac{d z^{2}}{z^{2}}+\frac{\eta_{\mu \nu} d x^{\mu} d x^{\nu}}{z^{2}}+\ldots
$$

where $f(z)=z^{2 / 3}\left(1-\left(z / R_{0}\right)^{4}\right)$ and $R_{0}$ is the position of the horizon[2. Although it was later found that the $S^{1}$ KK states do not strictly decouple in the interesting limits [18], we will use this background to study the interplay between the confining nature of gauge theory and its reggeization properties. Actually the qualitative arguments and approximations should be generic for most confining backgrounds? as already discussed in Ref. [15, 21].

In order to calculate the scattering amplitude, we have to evaluate the correlation function (2). Therefore we put the two tilted lines depicted in Fig. 1 on the boundary at $z=0$. Next we have to find the minimal surface in the appropriate geometry which has the two lines as its boundaries. The relative angle (tilt) in the $t-y$ plane and the separation in the transverse direction $x$ (impact parameter) therefore define the boundary conditions for the geodesic equations for the string.

As is well known for the Plateau problem of minimal surfaces 22] the boundary conditions determine the solutions. Although an exact solution for the minimal surface spanned by the tilted Wilson lines is unknown for the metric (5), the properties of the black hole ( $\mathrm{BH})$ geometry allow for quite a good approximation scheme.

Two salient features of the metric (5) are (i) the standard AdS prefactor $1 / z^{2}$ close to the boundary $(z=0)$, (ii) the existence of

\footnotetext{
${ }^{2}$ Compared to standard coordinates 13 we used $U=z^{-4 / 3}$ and $U_{T}=R_{0}^{-4 / 3}$.

3 Two other geometries for (supersymmetric) confining theories have been discussed recently $[19,20]$. They have the property that for small $z$, i.e. close to the boundary, the geometry looks like $A d S_{5} \times S^{5}$ (in [20] up to logarithmic corrections related to asymptotic freedom) giving a coulombic $q \bar{q}$ potential. For large $z$ the geometry is effectively flat. In all cases there is a scale, similar to $R_{0}$ above, which marks a transition between the small $z$ and large $z$ regimes.
} 


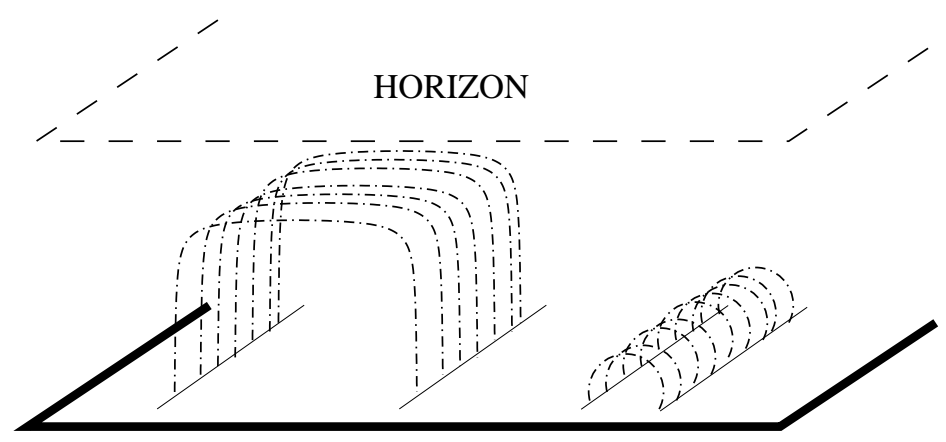

Boundary

Figure 2: The minimal surface in the black hole geometry. The Wilson lines are drawn here with vanishing angle of tilt $\theta=0$.

a horizon which limits from above the values of $z$. A consequence of (i) is that it is most efficient for a minimal surface to perform the "twisting" between the two Wilson lines as far away from the boundary as possible. Property (ii) effectively induces this twisting to occur near the horizon as we shall show below.

The appropriate minimal surface in the $\mathrm{BH}$ geometry will look as follows. Due to property (i), the minimal surface between well separated lines rises "vertically" in the $z$ direction up to the horizon without sizable motion in the other $\mathbb{R}^{4}$ coordinates (see a schematic representation in Fig. 2). The metric at the horizon is effectively flat

$$
d s_{\sim \text { horizon }}^{2} \sim \frac{1}{R_{0}^{2}}\left(\eta_{\mu \nu} d x^{\mu} d x^{\nu}\right)
$$

and the motion in the $z$ direction is "frozen out". Now near the horizon, following property (ii), the minimal surface performs the "twisting" (not displayed in Fig. 2) corresponding to the tilt angle $\theta$ between the initial Wilson lines. At this stage we thus have to find a minimal surface between the lines at an angle $\theta$ in the flat space metric (6). Finally the surface falls off again vertically towards the boundary. The area of the "vertical" pieces is removed by the standard subtractions [10], so the resulting area which enters the formula for the amplitude (2) may be approximated by the area of the "flat space" piece.

We will now substantiate this intuitive picture with a more quantitative study of the geodesic equation for the string. Let us determine under what conditions the minimal surface spanned by the two tilted 
Wilson lines is indeed predominantly flat and concentrated near the horizon following the general line of discussion of [15].

The minimal surface equations follow from the Nambu-Goto action.

$$
S=\frac{1}{2 \pi \alpha^{\prime}} \int_{-T}^{T} d \tau \int_{-l(\tau) / 2}^{l(\tau) / 2} \sqrt{\operatorname{det} h_{a b}}
$$

where the induced metric on the worldsheet is

$$
h_{a b} \equiv G_{i j} \frac{\partial X^{i}(\sigma, \tau)}{\partial v^{a}} \frac{\partial X^{j}(\sigma, \tau)}{\partial v^{b}} .
$$

$X^{i}$ stands for general coordinates $\left(z, x^{\mu}\right)$ in (5), $G_{i j}$ is the background metric, $v^{0} \equiv \sigma, v^{1} \equiv \tau$, and $l(\tau)=\sqrt{L^{2}+\theta^{2} \tau^{2}}$ is the euclidean distance between points on the two Wilson lines with the same value of the time coordinate $\tau$.

As a first remark we note that using the background metric (5) the terms in the induced metric $h_{\sigma \sigma}$ corresponding to the twisting are of the form

$$
\frac{1}{z^{2}}\left[\left(\frac{\partial y}{\partial \sigma}\right)^{2}+\left(\frac{\partial t}{\partial \sigma}\right)^{2}\right] .
$$

Hence, near the boundary $(z \rightarrow 0)$, the minimization will not change noticeably the twist angle. Thus the boundary conditions are "frozen" and transported to the vicinity of the horizon.

For further discussion we shall make an approximation (similar to [16]) of neglecting explicit $\tau$ dependence in the Euler-Lagrange equations following from (7) and leaving it only in the implicit dependence on the boundary conditions through $l(\tau)$. Within this approximation the estimate of [15], made for the case of the static $q \bar{q}$ potential may be directly applied to our problem.

In reference [15] a distance $d$ is defined, for all metrics giving confinement, which measures the transverse distance (on the boundary) over which the string worldsheet significantly deviates from being flat. In all cases the ratio $d / l(\tau) \longrightarrow 0$ when $l(\tau) \rightarrow \infty$.. Depending on the confining metric considered, $d$ behaves as a logarithm or a power of $l(\tau)$ smaller than one. It is interesting to note that the condition $d / l(\tau) \ll 1$ leads to a lower bound on the impact parameter $L$ since the above condition is most restrictive for the smallest value of $l(\tau)$, which is equal to $L$.

The precise dependence of $d(l(\tau))$ on the horizon scale $R_{0}$ depends on the metric considered. For instance for the metric (5) rescaling 
arguments lead to a dependence $d(l(\tau)) \sim R_{0}^{2 / 3} \log l(\tau)$. Therefore as long as the impact parameter is large with respect to $R_{0}$ the approximations considered in this section should be valid.

However, it may of course happen that the impact parameter distance between the two Wilson lines becomes much smaller than $R_{0}$. In this case (see Fig. 2) the minimal surface problem becomes less affected by the black hole geometry (or the large $z$ behaviour of the different metrics [19, 20]) and will just probe the small $z$ region of the geometry.

The precise behaviour at these shorter distances will depend on the type of gauge theory and, in particular, on the small $z$ limit of the appropriate metric. In this paper we will consider the generic case (from the $4 \mathrm{D}(\mathrm{S}) \mathrm{YM}$ point of view) when this limit resembles the original $A d S_{5} \times S^{5}$ geometry [1]. We will consider this conformal (non confining) regime in detail in a further section. We note that the same behaviour can be equivalently obtained through rescaling, by keeping the impact parameter fixed and putting the scale $R_{0} \rightarrow \infty$.

Let us concentrate in the following on the case when the impact parameter is larger than the scale $R_{0}$. To summarize the discussion, the string is then to a large degree concentrated in the region near the horizon (6) with the boundary conditions essentially transported from $z=0$. We are thus led first to calculate the area of the minimal surface bounded by the tilted lines in the flat geometry (6) at the horizon. We will first perform the calculation in euclidean signature and then perform the analytical continuation (4).

\section{Helicoid geometry}

The basic building block of our construction is a minimal surface spanned by two straight line segments of length $2 T$, corresponding to the two Wilson lines separated by a distance $L$ in the "transverse" direction $x$ and with a relative angle $\theta$ in the "longitudinal" plane:

$$
L_{1}: \tau \longrightarrow(\tau, 0,0,0) \quad L_{2}: \tau \longrightarrow(\tau \cos \theta, \tau \sin \theta, 0, L) .
$$

It is well-known that in the flat $\mathbb{R}^{4}$ geometry the minimal surface with infinite boudaries $\tau=-\infty \ldots+\infty$ is a helicoid. We will also be interested by the "truncated" helicoid where $\tau=-T \ldots T$.

\footnotetext{
${ }^{4}$ For finite cut-off $T$ in flat space, the truncated helicoid obviously remains a solution if one adds the boundary helices at $\tau=-T, T$ as new boundaries. Note, however, that
} 
Let us recall the minimal surface solution in flat space. The helicoid is the only regulated (spanned by straight lines) minimal surface. The truncated helicoid solution may be parametrized by

$$
\begin{aligned}
t & =\tau \cos \frac{\theta \sigma}{L} \\
y & =\tau \sin \frac{\theta \sigma}{L} \\
x & =\sigma
\end{aligned}
$$

where $\tau=-T \ldots T$ and $\sigma=0 \ldots L$ and $\theta$ is the total twisting angle.

Its area is given by the formula

$$
\begin{gathered}
\text { Area } \equiv S(T)=\int_{0}^{L} d \sigma \int_{-T}^{T} d \tau \sqrt{1+\frac{\tau^{2} \theta^{2}}{L^{2}}}= \\
=L T \sqrt{1+\frac{T^{2} \theta^{2}}{L^{2}}}+\frac{L^{2}}{2 \theta} \log \frac{\sqrt{1+\frac{T^{2} \theta^{2}}{L^{2}}}+\theta \frac{T}{L}}{\sqrt{1+\frac{T^{2} \theta^{2}}{L^{2}}}-\theta \frac{T}{L}} .
\end{gathered}
$$

Let us now perform the analytical continuation (4), which links euclidean correlation functions in gauge theories with minkowskian ones directly related to scattering amplitudes. A naive continuation of the area formula (12) leads to a pure phase factor in (2):

$$
\exp \left\{\frac{\sqrt{2 g_{Y M}^{2} N}}{2 \pi R_{0}^{2}} i\left[L T \sqrt{1+\frac{T^{2} \chi^{2}}{L^{2}}}+\frac{L^{2}}{2 \chi} \log \frac{\sqrt{1+\frac{T^{2} \chi^{2}}{L^{2}}}+\chi \frac{T}{L}}{\sqrt{1+\frac{T^{2} \chi^{2}}{L^{2}}}-\chi \frac{T}{L}}\right]\right\},
$$

where $1 / 2 \pi \alpha^{\prime}$ in (2) has been replaced by the factor $\sqrt{2 g_{Y M}^{2} N} /\left(2 \pi R_{0}^{2}\right)$ coming from the flat metric (6).

However the analytic structure of the euclidean area (12) involves cuts in the complex $T, \theta$ planes and thus leads to an ambiguity coming from the branch cut of the logarithm. In fact when performing the analytical continuation we have to specify the Riemann sheet of the logarithm (i.e. $\log \rightarrow \log +2 \pi i n$ ). This leads to an additional real multiplicative factor in (2):

$$
\exp \left\{-n \frac{\sqrt{2 g_{Y M}^{2} N}}{\chi} \frac{L^{2}}{2 R_{0}^{2}}\right\}
$$

with these boundaries the helicoid may be an unstable [23] minimum for a too large value of the cut-off. We will not consider this problem in the present paper. 
the form of which is uniquely fixed by the euclidean expression (12) up to a choice of the integer $n$. Within the classical approximation which we have been using it is not possible to determine the value of $n$. On a more physical ground, in section 4, we will relate the analogue of the label $n$ which appears in the calculation of Wilson loop correlators with multivalued saddlepoint minima of a minimization equation and thus to different classical solutions. The determination of the relative weights of the various contributions goes beyond the classical approximation used throughout this paper?.

As can be seen the contribution (14) is cut-off independent.

Another useful way of deriving the above factor (14) can be directly obtained from the integral leading to (12). This method can be generalized to more complicated background geometries, for instance to the conformal case, which we will consider later, for which we lack an exact expression of the form (12).

Let us perform only the first part of the analytical continuation (4) $\theta \longrightarrow-i \chi$ but otherwise remain with the time variable $T$ in Euclidean space. This procedure yields the expression:

$$
\int_{-\frac{L}{\chi}}^{\frac{L}{\chi}} d \tau \int_{0}^{L} d \sigma \sqrt{1-\frac{\tau^{2} \chi^{2}}{L^{2}}}=\frac{\pi L^{2}}{2 \chi} .
$$

We see that the imaginary part may be obtained by integrating ( $n$ times) around the branch cut of the square root. A convenient reinterpretation of the above formula follows from performing the change of variables $\sigma \longrightarrow \sigma^{\prime}=\sigma \sqrt{1-\frac{\tau^{2} \chi^{2}}{L^{2}}}$. Then we get

$$
2 i n \int_{-\frac{L}{\chi}}^{+\frac{L}{\chi}} d \tau \int_{0}^{\sqrt{1-\frac{\tau^{2} \chi^{2}}{L^{2}}} L} d \sigma^{\prime}=n i \pi \frac{L^{2}}{\chi}
$$

which is effectively twice $(\times i n)$ the area of a minimal surface bounded by a 'half-elipse' of radii $L$ and $L / \chi$. This $T$-independent imaginary part is unaffected by the second part of the analytical continuation (41), and leads directly to the factor (14).

\footnotetext{
${ }^{5}$ We also note the close similarity of the $n, L$ and $\chi$ dependence in (14) with an analogous factor $\exp \left(-n L^{2} / \pi \chi\right)$ in the imaginary part of the $\mathrm{D}$ brane scattering amplitude [24] where $n$ labels the poles of the appropriate string partition function between the branes.
} 


\section{Reggeization in quark-(anti)quark scattering}

Our result for the Wilson line correlation function for the $A d S \mathrm{BH}$ geometry gives rise to the following contributions

$$
\begin{aligned}
\tilde{A}_{n}= & \exp \left\{\frac{\sqrt{2 g_{Y M}^{2} N}}{2 \pi R_{0}^{2}} i\left[L T \sqrt{1+\frac{T^{2} \chi^{2}}{L^{2}}}+\frac{L^{2}}{2 \chi} \log \frac{\sqrt{1+\frac{T^{2} \chi^{2}}{L^{2}}}+\chi \frac{T}{L}}{\sqrt{1+\frac{T^{2} \chi^{2}}{L^{2}}}-\chi \frac{T}{L}}\right]\right\} \\
& \times \exp \left\{-n \frac{\sqrt{2 g_{Y M}^{2} N}}{\chi} \frac{L^{2}}{2 R_{0}^{2}}\right\} .
\end{aligned}
$$

There is a divergent phase in the above amplitude when the temporal length of the lines $T$ goes to infinity. We interpret this divergence as reflecting the expected IR divergence of the $q-\bar{q}$ scattering amplitude [7, 8]. A consistent way to eliminate this cut-off dependence is to consider an IR finite physical quantity like scattering of two $q \bar{q}$ pairs (see section 4). In the present case of Wilson lines, the specific factorized form of (17) allows for a determination of an IR finite contribution, which can be interpreted as an effect of inelastic channels on the Wilson line correlator.

It is known since a very long time that the superposition of long range and short range potentials in the Schroedinger equation leads to a factorization formula for the relevant $\mathrm{S}$ matrix elements for each partial wave [25]. For instance in nuclear physics, the superposition of long range coulombic and short range interactions leads to a factorization into the elastic coulombic S matrix element and a short range amplitude modified by the long-range background. The elastic S matrix may be treated as a redefinition of the asymptotic initial and final states. The amplitude reads

$$
A(l, s)=e^{2 i \delta(l, s)} \cdot T(l, s)
$$

where $\delta$ is the real phase shift due to the elastic long range interactions

and $T$ is the short range part of the amplitude. For instance in the QED result for electron scattering [26], the real phase shift exhibits a divergence which can be written as

$$
e^{2 i \delta(l, s)} \propto \exp \left\{i \frac{e^{2}}{4 \pi} \operatorname{coth} \chi \log \left(\frac{L^{2}}{4 T^{2}}\right)\right\}
$$

where $1 / T$ has been substituted for a fictitious photon mass (IR regulator). 
In hadronic interaction physics [27, a similar factorization appears for the S matrix elements for 2-body channels in terms of an elastic contribution and an amplitude $T(l, s)$ which, by unitarity of the $\mathrm{S}$ matrix, arises from the contribution of many inelastic channels to the 2-body $\mathrm{S}$ matrix [28]. In this context the amplitude (18) can be related to the inelasticity (overlap matrix) in the scattering namely

$$
T(l, s)=\frac{1-\sqrt{1-2 f(l, s)}}{2}
$$

where the overlap matrix elements $f(l, s)$ are defined from the 2-body S matrix contribution to unitarity $|S(l, s)|^{2} \equiv 1-2 f(l, s)$.

We are led to interpret our resulting amplitude (17) in the same way. The factor (13) can be treated as redefining the initial and final $q \bar{q}$ states due to long range interactions? Naturally this phase is IR divergent. The analogous inelastic contribution $T_{n}(l, s)$ is obtained to be the cut-off independent factors (14). Note that this physical interpretation requires the integer $n$ to be positive. We will return to the discussion of the $n$ dependence in a further section.

Let us discuss both factors of the amplitude (17). The contribution of the real phase shifts behaves in the large $T$ limit like

$$
\exp \left\{\frac{\sqrt{2 g_{Y M}^{2} N}}{2 \pi R_{0}^{2}} i\left(T^{2} \chi+\frac{L^{2}}{\chi} \log \left(\frac{2 \sqrt{e} \chi T}{L}\right)\right)+O\left(1 / T^{2}\right)\right\}
$$

The appearance of the IR divergent $T^{2}$ and $L^{2} \log T$ terms in the phase shift can be linked with the linear confining potential of the theory.

The effect of the confining potential is expected to generate inelastic channels through the phenomenon of string breaking and/or closed string emission. Within the above framework, where we select initial and final $q \bar{q}$ states, this contribution is expected to appear as an inelastic real factor in the amplitude, while the phase factor diverges with $T \longrightarrow \infty$.

The inelastic $q \bar{q}$ interaction amplitude at level $n$ is

$$
T_{n}(l, s)=\exp \left\{-n \frac{\sqrt{2 g_{Y M}^{2} N}}{\chi} \frac{L^{2}}{2 R_{0}^{2}}\right\}
$$

\footnotetext{
${ }^{6}$ It is clear that there remains a freedom in attributing a finite real phase shift either to the redefinition of the states or to the interaction. Here we adopt the convention that $T(l, s)$ is purely real and thus contains information only on the inelasticity.
} 
where the initial and final states are both $q \bar{q}$. It can be easily fourier transformed into transverse momentum space giving

$$
T_{n}(s, t)=\frac{i R_{0}^{2} \ln s}{n \sqrt{2 g_{Y M}^{2} N}} s^{1+\frac{R_{0}^{2}}{2 n{\sqrt{2 g_{Y M}^{2}}}^{2}} t} .
$$

This contribution is thus reggeized with a linear Regge trajectory with unit intercept and the slope given by the string tension related to the horizon distance $R_{0}^{2}$.

It is worthwhile to consider what changes in the preceeding discussion if we go from $q-\bar{q}$ scattering to $q-q$ scattering. In geometric terms, this corresponds to changing the orientation of one of the lines, and since the string worldsheet spanned on the Wilson lines is oriented, the twisting angle of the helicoid changes as

$$
\theta \longrightarrow \theta-\pi
$$

Upon analytical continuation this means that $\chi \sim \log s$ changes to $\chi-i \pi \sim \log s e^{-i \pi}$, as required by crossing properties, which are seen to have here a very simple geometric interpretation. We note that in the asymptotically high energy $\operatorname{limit} \log s \gg 1$, one obtains the same factors (17) for both $q q$ and $q \bar{q}$ channels. Keeping the next to leading correction corresponding to $\log s \longrightarrow \log s e^{-i \pi}$ preserves the crossing relations between those channels.

Finally let us compare our result with the general structure of Wilson line correlators at weak gauge coupling. Indeed, the large $T$ dependence of the $q \bar{q}$ amplitudes we discuss reflects IR divergences which appear already in perturbative (weak coupling) calculations of the same quantities.

For instance in the case of QED the whole dynamics is contained in the infinite phase factor (19) and the divergence is logarithmic.

The (renormalon improved) 1-loop QCD result [8, 29] for $q \bar{q}$ scattering is

$$
\exp \left\{-\frac{1}{\chi} \frac{\alpha_{s}}{\pi} \log \left(\frac{T}{L}\right)-\frac{\rho}{\pi} \Lambda^{2} \frac{L^{2}}{\chi}\right\}
$$

where $\rho$ is an undetermined nonperturbative parameter. We note the compatibility between the nonperturbative cut-off independent piece in (25) and an analogous term in our result (22). Our nonperturbative result gives a hint on the scale and coupling dependence.

If the intercept one common to all contributions $T_{n}(s, t)$ (see formula $(23))$ is not spoiled by the different weights corresponding to 
fluctuations of the worldsheet around classical solutions, it would be a candidate for the intercept one trajectories (the so-called pomeron and odderon) which are expected to emerge from a confining strongly interacting gauge theory.

\section{Conformal case}

The flat metric approximation which we have used to derive the resulting area (12) assumed that the impact parameter $L$ is sufficiently large with respect to the scale set by the horizon radius $R_{0}$ (or a similar scale in the backgrounds [19, 20] interpolating between a confining geometry at large $z$ and approximately $A d S_{5} \times S^{5}$ near the boundary $z=0)$. In this regime the dominant contribution to the amplitudes came from the part of the string worldsheet stretched near the horizon.

If we go to smaller impact parameters $L<R_{0}$ (and also for $T<$ $R_{0}$ ), the minimal surface would only penetrate into a limited region near the boundary $z=0$, see Figure 2 . In the scenarios which behave better at short distances than the original $\mathrm{BH}$ proposal, the metric becomes closer and closer to the conformal $A d S_{5}$ case. We note that the $A d S_{5} \times S^{5}$ setting is directly related to scattering in the $\mathcal{N}=4$ SYM. This different geometry leads to a qualitatively new behaviour which we now analyze.

\section{The conformal $A d S_{5}$ case}

In the case of $\mathcal{N}=4 \mathrm{SYM}$ corresponding to the $A d S_{5} \times S^{5}$ background we do not know yet the exact generalization of the helicoid, and some approximation scheme is needed. As in the previous case, we will concentrate on extracting the inelastic contribution which appears also

here to be independent of the IR temporal cut-off $T$. We use the method outlined in section 2 leading to formulae (15)-(16).

Within a variational approximation approach, we will look for a minimal solution in a restricted set of surfaces ("generalized helicoids") parameterized by

$$
\begin{aligned}
t & =\tau \cos \frac{\theta \sigma}{L} \\
y & =\tau \sin \frac{\theta \sigma}{L} \\
x & =\sigma
\end{aligned}
$$




$$
z=z(\sigma, \tau) .
$$

Evaluation of the induced metric gives rise to the following area functional:

$$
\int_{-T}^{T} d \tau \int_{0}^{L} d \sigma \frac{1}{z^{2}} \sqrt{\left(1+\frac{\tau^{2} \theta^{2}}{L^{2}}\right)\left(1+z_{\tau}^{2}\right)+z_{\sigma}^{2}}
$$

We perform a change of variables $\sigma \longrightarrow \sigma^{\prime}=\sigma \sqrt{1+\frac{\tau^{2} \theta^{2}}{L^{2}}}$ which yields

$$
\frac{1}{2 \pi \alpha^{\prime}} \int_{-T}^{T} d \tau \int_{0}^{L \sqrt{1+\frac{\tau^{2} \theta^{2}}{L^{2}}}} d \sigma^{\prime} \frac{1}{z^{2}} \sqrt{1+z_{\tau}^{2}+z_{\sigma^{\prime}}^{2}} .
$$

As in the previous section the cut-off independent part is obtained from the branch cut structure of the area functional (31). The analytic continuation $\theta \longrightarrow-i \chi$ changes the boundary conditions for the minimal surface to be a half elipse of width $L / \chi$ and height $L$ (the upper integration limit in (31) then becomes $L \sqrt{1-\tau^{2} \chi^{2} / L^{2}}$ ). Due to conformal invariance we know that the minimal area has the following form:

$$
A_{\text {minimal }}=f(L / \epsilon, \chi)+g(\chi)
$$

where $\epsilon$ is the " $5^{\text {th} " ~ A d S ~ c o o r d i n a t e ~ w h e r e ~ w e ~ p u t ~ t h e ~ D 3 ~ b r a n e ~ p r o b e . ~}$ $\epsilon$ translates directly into the mass of the $W$ bosons which play here the role of quarks. We do not expect higher poles in $\epsilon$ than first order, which are in the standard way subtracted out [10, 30], so we have at most a logarithmic behaviour in $L / \epsilon$.

It is possible to obtain an approximate result in the high energy $\chi \longrightarrow \infty$ limit from known properties of Wilson loop expectation values [10, 30]. The half-elipse has two cusps each with an angle $\pi / 2$, whose contribution to $A_{\text {minimal }}$ can be obtained from the results of [30]. This leads to the following logarithmic terms:

$$
-2 \frac{1}{2 \pi} F(\pi / 2) \cdot \log \frac{L}{\epsilon \chi}
$$

where $F(\Omega)$ is a complicated function calculated in [30] $(F(\pi / 2) \sim$ $0.3 \pi)$. The $\epsilon$ independent term $g(\chi)$ in (32) can be approximated by noting that at high energies the half-elipse is very much elongated and looks like parallel lines of length $L$, roughly $2 L / \chi$ apart. An approximate evaluation is then given by integrating the coulombic potential [10]:

$$
-c \cdot \int_{0}^{L} \frac{d \sigma^{\prime}}{\frac{2}{\chi} \sqrt{L^{2}-\sigma^{\prime 2}}}=-c \frac{\pi}{4} \cdot \chi
$$


where $c=8 \pi^{3} / \Gamma^{4}(1 / 4)$ is the coefficient in front of the (screened) coulombic potential. So we get

$$
T_{n}(l, s) \sim\left(\frac{L}{\epsilon \log s}\right)^{n \frac{F(\pi / 2)}{\pi} \frac{\sqrt{2 g_{Y M^{N}}^{2}}}{2 \pi}} s^{n \frac{2 \pi^{4}}{\Gamma(1 / 4)^{4}} \frac{\sqrt{2 g_{Y M^{N}}^{2}}}{2 \pi}} .
$$

Here, as in the case of the confining theory, the values of $n$ and the weights of the different components $T_{n}(l, s)$ are not specified.

Let us comment on the behaviour of the various components. In all cases we obtain a factorized energy behaviour with no moving Regge trajectories. We note that for $n$ positive a similar energy dependence (i.e. with intercept greater than 1 and a (nearly) flat Regge trajectory) is obtained by resumming the leading $\log s$ terms in the perturbative expansion at weak coupling [31] for the singlet exchange amplitude. In the conformal case, there remains a non-perturbative screening effect (already present for the static $q \bar{q}$ potential [10]) which appears as the change $g_{Y M}^{2} N \rightarrow \sqrt{g_{Y M}^{2} N}$ in the exponent of $s$.

Considering the impact parameter dependence and its Fourier transform to momentum space, in the window of convergence (the exponent of $L$ in (35) between -2 and $-3 / 2$ ), we get

$$
T_{n}(s, t) \sim i s^{1+n \frac{2 \pi^{4}}{\Gamma(1 / 4)^{4}} \cdot \frac{\sqrt{2 g_{Y M^{N}}^{2}}}{2 \pi}}\left(\frac{1}{t}\right)^{1+n \frac{F(\pi / 2)}{2 \pi} \frac{\sqrt{2 g_{Y M}^{2} N}}{2 \pi}}
$$

Otherwise one observes either an UV divergence (for exponent values less than -2 ) or an IR one (for values larger than $-3 / 2$ ). For positive values of $n$ the IR divergence requires a careful treatment which is beyond the scope of this paper. Note that, in the case of $\mathcal{N}=4 \mathrm{SYM}$, it can lead to infra-red divergent pieces also in the inelastic amplitude, as is the case already in the perturbative limit [8, 32].

\section{Conformal/non-conformal transition}

As already mentioned, the result (36) obtained for the pure $A d S_{5} \times S^{5}$ case should give the dominant behaviour also for the confining theory for impact parameters small with respect to the horizon scale] $R_{0}$ (or more generally an analogous transition scale in the geometries [19, 20]). Indeed this $R_{0}$ provides a natural value of the impact parameter cut-off $L_{0}$. Thus even in the confining theory, when the impact

\footnotetext{
${ }^{7}$ We need also a sufficiently small $T$ parameter.
} 
parameter is decreased and gets smaller than $R_{0}$, we expect a transition from the set of components (22) to the results (35), as long as the relevant geometry for small $z$ is similar to $A d S_{5} \times S^{5}$.

This process can be observed by noting that both (22) and (35) were derived from a minimal surface spanned on a semielipse. The result for impact parameters $L \geq O\left(R_{0}\right)$ was obtained by using the area law for Wilson loops, while the conformal case (corresponding here to $L \ll R_{0}$ ) used an approximation using coulombic potential. The solution of the appropriate minimal surface problem in the full geometry would lead to an interpolation between the two extreme cases.

\section{Wilson loop correlators and scatter- ing amplitudes}

We saw that an inherent feature of the $q-\bar{q}$ scattering amplitude is its IR divergence. In order to remedy this, and also to show a context where the finite behaviour of the inelastic amplitudes calculated in the previous section appears directly without the infinite phases, we are led to consider the scattering of two $q \bar{q}$ pairs of transverse size $a$, and impact parameter distance $L$. This process is interesting to study in itself, since it gives some information on the scattering amplitudes between colourless states in gauge theories at strong coupling.

For this setup we have to calculate the correlation function of two Wilson loops [9], where the loops are choosen to be elongated along the "time" direction and have a large but arbitrary temporal length $T$ (the exact analogue for Wilson loops of $T$ considered in the previous section). However, the cut-off dependence on $T$ is expected to be removed together with the related IR divergence which was present for the case of Wilson lines.

For large positive and negative times the minimal surface will be well approximated by two seperate copies of the standard minimal surfaces for each loop separately. When we come to the interaction region, and for $L$ sufficiently small, one can lower the area by forming a "tube" joining the two worldsheets. Since we want to calculate the normalized correlator $\left\langle W_{1} W_{2}\right\rangle /\left\langle W_{1}\right\rangle\left\langle W_{2}\right\rangle$, the contributions of the regions outside the tube will cancel out (in a first approximation

neglecting deformations near the tube). Therefore we have just to find the area of the tube, and subtract from it the area of the two 
independent worldsheets. It is at this stage that we see that the result does not depend on the maximal length of the Wilson loops $T$, and hence is IR finite. The whole contribution to the amplitude will just come from the area of the tube.

Since we cannot obtain an exact minimal surface for these boundary conditions, let us perform a variational approximation. Namely we will consider a family of surfaces forming the tube, parameterized by $T_{\text {tube }}$, which has the interpretation of an "effective" time of interaction. Then we will make a saddle point minimization of the area as a function of this parameter.

Suppose that the tube linking the two Wilson lines is formed in the region of the time parameter $t \in\left(-T_{\text {tube }}, T_{\text {tube }}\right)$. In our approximation its two "sides" are formed by sheets of the helicoid solution (of area $S\left(T_{\text {tube }}\right)$, see (12) for the euclidean case and (13) for the minkowskian one). The front and back will be each approximated by strips of area $a L \sqrt{1+\frac{T_{t u b e}^{2} \theta^{2}}{L^{2}}}\left(\right.$ we assume $a, L \geq R_{0}$ ).

The total area corresponding to the two Wilson loops is then given by

$\operatorname{Area}\left(T_{\text {tube }}\right)=2 L \int_{-T_{\text {tube }}}^{T_{\text {tube }}} d \tau \sqrt{1+\frac{\tau^{2} \theta^{2}}{L^{2}}}+2 a L \sqrt{1+\frac{T_{\text {tube }}^{2} \theta^{2}}{L^{2}}}-4 a \cdot T_{\text {tube }}$,

where $-2 a T_{\text {tube }}$ is the contribution of each individual Wilson loop to the normalization $1 /\left\langle W_{1}\right\rangle\left\langle W_{2}\right\rangle$ of the Wilson loop correlation function.

Analytically continuing the area formula (37) to the Minkowskian case and using a convenient change of variables, the Minkowskian area can be put in the following simple form

$$
\operatorname{Area}\left(T_{\text {tube }}\right)=\frac{2 L^{2}}{\chi}\left\{\phi+\frac{\sin 2 \phi}{2}+\rho \chi \cos \phi-2 \rho \sin \phi\right\}
$$

where $\rho \equiv a / L$ and $\sin \phi=i \chi T_{\text {tube }} / L$ is the new variational parameter.

In the strong coupling limit $\left(\alpha^{\prime}=1 / \sqrt{2 g_{Y M}^{2} N} \rightarrow 0\right)$ the parameter $\phi$ is dynamically determined from the saddle point equation:

$$
0=\frac{\partial \operatorname{Area}(\phi)}{\partial \phi}=\cos \phi(\cos \phi-\rho)-\frac{\rho \chi}{2} \sin \phi
$$

It is easy to realize that for large enough energy, there exists a solution 
with $\phi \sim \pm n \pi$. Inserting this solution into the area (38) we find

$$
\operatorname{Area}(\phi)=-\frac{2 L^{2}}{\chi} n \pi+2 a L(-1)^{n}
$$

where we retain the physical solutions with $n$ positive integer. We thus find a set of solutions very similar to the inelastic factor obtained in section 2. The modification due to the front-back contribution $2 a L$ is negligible in the Fourier transformed amplitude for momentum transfer $\sqrt{-t} \gg a / R_{0}^{2}$. Also this term is probably more dependent on the treatment of the front-back parts of the tube in our approximation.

It is interesting to note that the minimization (39) gives rise in a natural way to a similar set of solutions parameterized by integers as found from the branch cut arguments in section 2 . Each value of $n$ corresponds to a saddle point i.e. a classical solution. The determination of the weights of each component to the total scattering amplitude is beyond the reach of the classical approximation.

For completeness, let us briefly discuss the general saddle point solution. For lower energies, there are families of solutions also leading to reggeized behaviour but with distorted trajectories. For $\chi$ small there exist solutions with $\phi$ imaginary and thus leading to elastic parts of the amplitude. The study of these solutions is beyond the scope of the present paper. For too large impact parameters we may enter the purely elastic regime found in [5] which does not correspond to connected minimal surfaces (the Gross-Ooguri transition [14).

As a word of caution (and incentive for further study) we note that the saddle point in terms of $T_{\text {tube }}$ is mainly driven to complex values. This indicates that a complete treatment and an investigation of the Gross-Ooguri transition requires a more refined study of the tube minimal surface.

Let us analyze the properties of the resulting amplitude. Recalling that charge conjugation acting on one of the $q \bar{q}$ pairs is equivalent to considering the transformation $\chi \rightarrow \chi-i \pi$, it is convenient to analyze the components of definite signature [3] with the even and odd contributions given by

$$
\tilde{T}_{n}^{ \pm}(l, s)=e^{-n \frac{\sqrt{2 g_{Y M}^{2}}}{\chi} \frac{L^{2}}{R_{0}^{2}}} \pm e^{-n \frac{\sqrt{2 g_{Y M}^{2}}}{\chi-i \pi} \frac{L^{2}}{R_{0}^{2}}} .
$$

Note the relative factor of 2 in the exponent in comparison with (22) due to the two sheet structure of the minimal surface. 
Using the Fourier transform (1) we finally get

$$
T_{n}^{ \pm}(s, t)=\frac{i R_{0}^{2} \ln s}{2 n \sqrt{2 g_{Y M}^{2} N}} s^{\alpha_{n}(t)} \mp \frac{i R_{0}^{2} \ln (-s)}{2 n \sqrt{2 g_{Y M}^{2} N}}(-s)^{\alpha_{n}(t)},
$$

where

$$
\alpha_{n}(t)=1+\frac{R_{0}^{2}}{4 n \sqrt{2 g_{Y M}^{2} N}} t .
$$

Let us consider the contribution with $n=1$ which is dominant at large $L$. It is easy to realize that the amplitude (42) corresponds to specific Regge singularities in the S-matrix framework, namely double Regge poles whose trajectory is given by $\alpha_{1}(t)$. Indeed, using the usual Mellin transform $s^{\alpha} \equiv \int \frac{s^{j} d j}{2 i \pi(j-\alpha)}$, it can be written in the following equivalent forms:

$$
\begin{aligned}
T_{1}^{ \pm}(s, t) & =\frac{i R_{0}^{2}}{2 \sqrt{2 g_{Y M}^{2} N}} \frac{\partial}{\partial \alpha}\left\{s^{\alpha_{1}(t)} \mp(-s)^{\alpha_{1}(t)}\right\} \\
& =\frac{R_{0}^{2}}{2 \sqrt{2 g_{Y M}^{2} N}} \int_{\mathcal{C}} \frac{d j}{\pi} \frac{e^{-i \pi j / 2} s^{j}}{\left(j-\alpha_{1}(t)\right)^{2}}\left\{\begin{array}{c}
i \sin \left(\frac{\pi j}{2}\right) \\
\cos \left(\frac{\pi j}{2}\right)
\end{array}\right\},
\end{aligned}
$$

where the complex contour $\mathcal{C}$ can be taken around the Regge (di)pole trajectory $\alpha_{1}(t)$ and the signature factors are either $\sin \pi j / 2$ or $-i \cos \pi j / 2$ depending on the positive or negative signature.

Let us discuss the contributions $T_{n}$ to the amplitude with $n>1$. In the absence of a direct determination of their relative weights, it is interesting to note that unitarization of Regge amplitudes in the S matrix framework 28, 27] leads to a similar decomposition where the $T_{n}$ correspond to Regge pole/cut singularities. In particular, the overlap matrix formalism [28], see (20), leads to a specific model for the relative weights of the $T_{n}$ 's, if we assume a gaussian distribution $f(l, s) \sim f_{0} \exp \left(-\frac{\sqrt{2 g_{Y M}^{2} N}}{\chi} \frac{L^{2}}{2 R_{0}^{2}}\right)$ for the inelasticity. In this framework [28] unitarity is fulfilled whenever $0<f_{0}<1 / 2$. However the derivation of the Wilson line/loop correlation function does not allow us to give model-independent predictions for these weights in the total amplitude.

Finally let us comment on the relation of our results on the trajectory $\alpha(t)$ with the glueball spectrum calculations [33]. An extrap- 
olation of the trajectory (43) to positive $t$ leads to masses of the form

$$
M^{2}=4 n(J-1) \frac{\sqrt{2 g_{Y M}^{2} N}}{R_{0}^{2}}
$$

where $J$ is the spin and $n$ labels the different trajectories. Because of the appearance of coupling constant dependence it is easy to see that these states correspond to massive string states and not to supergravity fields associated with the glueballs found in [33]. Indeed the latter states have masses proportional just to $1 / R_{0}^{2}$ and spin limited by $J \leq 2$. The appearence of massive string states is not surprising in our case as we consider an extended string worldsheet between the two Wilson loops instead of a supergravity field exchange. The transition between both situations and thus the relation between both sets of states remains an open problem.

We should note that our approximations for calculating the Wilson loop correlator (which is the channel relevant for glueballs) are rather crude and become problematic at small $t$ (consider the discussion after (40)). Therefore the extrapolation of the linear trajectory into the glueball regime can easily break down. Unfortunately the complexity of the minimal surface problem with the Wilson loop boundary conditions does not allow us to make more quantitative estimates.

\section{Conclusions and outlook}

Let us give our main conclusions. By computing Wilson line and Wilson loop correlation functions in the framework of the AdS/CFT correspondence we show a relation between minimal surface problems in $A d S_{5}$ metrics and reggeization in gauge field theory at strong coupling.

For Wilson line correlators, we isolate in certain cases IR finite inelastic amplitudes coming from the branch cut structure of the analytical continuation of helicoid-like surfaces i.e. minimal surfaces with straight line boundary conditions corresponding to classical trajectories in Minkowski space.

We considered three cases: (i) flat metric approximation of an $A d S$ black hole metric giving rise to Regge amplitudes with linear trajectories, (ii) an approximate evaluation for the conformal $A d S_{5} \times$ 
$S^{5}$ geometry leading to flat Regge trajectories $\$$ and (iii) evidence for a transition, in a confining theory, from behaviour of type (i) to (ii) when the impact parameter decreases below the interpolation scale set by the horizon radius. In this case, confinement provides a natural IR cut-off scale.

In a second stage we considered the correlation function of two Wilson loops elongated along the light cone directions for the confining geometry. This configuration corresponds to a high energy scattering amplitude between colourless $q \bar{q}$ states. We use a variational approximation where the minimal surface is constructed from two helicoidal sheets. As expected, the obtained amplitude is free from IR divergences and gives rise to reggeization with a linear trajectory with unit intercept. For high energies the amplitude is imaginary and thus mainly reflects the inelasticity of the process.

These results call for some comments.

We note that the structure of our resulting amplitudes for the confining case (in particular the $n, \chi$ and $L$ dependence) matches the calculations of the imaginary part of flat space D-brane scattering amplitudes [24] and some specific Wilson loop correlators [1] [34, when the "effective" string length $\sqrt{\alpha^{\prime}}$ is taken to be set by the horizon radius in our case. It is interesting to note that the imaginary part in those calculations is generated from the singularities of the string amplitudes which are an infinite set of poles. The slopes of the trajectories are the same as in our case (43), while the intercepts are different. However the geometrical configurations in [34] is quite different from the one we considered in section 4. Even in the flat space approximation it would be useful to have a direct string calculation of the tube configuration.

Beyond the flat space approximation, we want to emphasize the interest of solving exactly the well defined mathematical problem of finding the generalization of the helicoid for various AdS metrics, i.e. the minimal surface spanned between infinite lines forming an angle $\theta$ at the boundary. Another goal is to go beyond the classical approximation in order to derive the $n$-dependent weights to the scattering amplitudes.

Indeed the generalization of the helicoidal geometry in AdS space seems to be a building block for high energy scattering amplitudes in

\footnotetext{
${ }^{8} \mathrm{~A}$ remaining IR divergence in the inelastic amplitude is still present in the absence of confinement.

${ }^{9}$ We have extracted the imaginary part from the formulae in Ref. [34], along the lines of 24 .
} 
gauge theories at strong coupling.

\section{Acknowledgements}

RJ was partially supported by KBN grants 2P03B00814, 2P03B08614. We thank T. Garel and B. Giraud for useful remarks.

\section{References}

[1] J. Maldacena, Adv. Theor. Math. Phys. 2 (1998) 231;

S.S. Gubser, I.R. Klebanov and A.M. Polyakov, Phys. Lett. B428 (1998) 105;

E. Witten, Adv. Theor. Math. Phys. 2 (1998) 253.

[2] O. Aharony, S.S. Gubser, J. Maldacena, H. Ooguri and Y. Oz, Large $N$ field theories, String Theory and Gravity, hepth/9905111.

[3] M. Froissart and R. Omnes, Mandelstam Theory and Regge Poles, Frontiers in Physics, Benjamin ed. 1963.

[4] M.Froissart, Phys.Rev. 123 (1961) 1053.

[5] R.A. Janik and R. Peschanski, Nucl. Phys. B565 (2000) 193; R.A. Janik, Gauge Theory Scattering from the AdS/CFT correspondence, Cargese summer school 1999, hep-th/9909124.

[6] O. Nachtmann, Ann. Phys. 209 (1991) 436.

[7] H. Verlinde and E. Verlinde, QCD at High Energies and TwoDimensional Field Theory, hep-th/9302104.

[8] G.P. Korchemsky, Phys. Lett. B325 (1994) 459.

[9] O. Nachtmann, High Energy Collisions and Nonperturbative $Q C D$, hep-ph/9609365 (see e.g. eq. (3.87) for colourless state scattering).

[10] J. Maldacena, Phys. Rev. Lett. 80 (1998) 4859;

S.-J. Rey and J. Yee, Macroscopic strings as heavy quarks in large $N$ gauge theory and anti-de Sitter supergravity, hep-th/9803001.

[11] E. Witten, Adv. Theor. Math. Phys. 2 (1998) 505.

[12] S.-J. Rey, S. Theisen and J.-T. Yee, Nucl. Phys. B527 (1998) 171

[13] A. Brandhuber, N. Itzhaki, J. Sonnenschein and S. Yankielowicz, Phys. Lett. B434 (1998) 36 
[14] D.J. Gross and H. Ooguri, Phys. Rev. D58 (1998) 106002.

[15] Y. Kinar, E. Schreiber and J. Sonnenschein, Nucl. Phys. B566 (2000) 103; see also section 11 in 21.

[16] M. Rho, S.-J. Sin and I. Zahed, Phys. Lett. B466 (1999) 199.

[17] E. Meggiolaro, Z. Phys. C76 (1997) 523, , Eur. Phys. J. C4 (1998) 101, , Phys. Rev. D53 (1996) 3835.

[18] H. Ooguri, H. Robins and J. Tannenhauser, Phys. Lett. B437 (1998) 77

[19] A. Kehigas and K. Sfetsos, Phys. Lett. B456 (1999) 22

[20] C. Angelantonj and A. Armoni, RG Flow, Wilson Loops and the Dilaton Tadpole, hep-th/0003050

[21] Y. Kinar, E. Schreiber, J. Sonnenschein and N. Weiss, Quantum fluctuations of Wilson loops from string models, hep-th/9911123.

[22] A.T. Fomenko, The Plateau Problem Part 1 and 2, GordonBreach 1989

[23] A. Boudaoud, P. Patrìcio, and M. Ben Amar, Phys. Rev. Lett. 83 (1999) 3836.

[24] C. Bachas, Phys. Lett. B374 (1996) 37.

[25] E.g. see A. Messiah, Quantum Mechanics, vol I and II North Holland 1961.

[26] H. Cheng and T.T. Wu, Phys. Rev. Lett. 22 (1969) 666;

H. Abarbanel and C. Itzykson, Phys. Rev. Lett. 23 (1969) 53.

[27] A. Białas and L. van Hove, Nuovo Cim. 38 (1965) 1385

[28] L. van Hove, Rev. Mod. Phys. 36 (1964) 655

[29] I.A. Korchemskaya, Nucl. Phys. B490 (1997) 306

[30] N. Drukker, D.J. Gross and H. Ooguri, Phys.Rev., D60 (1999) 125006;H. Ooguri, Prog.Theor.Phys.Suppl., 134 (1999) 153.

[31] L.N. Lipatov, Sov. J. Nucl. Phys. 23 (1976) 642; V.S. Fadin, E.A. Kuraev and L.N. Lipatov, Phys. Lett. B60 (1975) 50; E.A. Kuraev, L.N. Lipatov and V.S. Fadin, Sov. Phys. JETP 44 (1976) 45, 45 (1977) 199; I.I. Balitsky and L.N. Lipatov, Sov. J. Nucl. Phys. 28 (1978) 822.

For a discussion in impact parameter space see G.P.Salam, Quarkonium Scattering at High Energies, PhD thesis, University of Cambridge 1996. 
[32] Z. Bern, J. Rozowsky and B. Yan, Two loop $\mathcal{N}=4$ Supersymmetric Amplitudes and QCD, hep-ph/9706392, talk given at DIS 97.

[33] C. Csaki, H. Ooguri, Y. Oz and J. Terning, JHEP 9901 (1999) 017; R. de Mello Koch, A. Jevicky, M. Mihailescu and J.P. Nunes, Phys. Rev. D58 (1998) 105009.

For a recent extension to spin two glueballs see R.C. Brower, S.D. Mathur and C.-I Tan, Glueball Spectrum for QCD from AdS Supergravity Duality, hep-th/0003115; N.R. Constable and R.C. Myers, JHEP 9910 (1999) 037.

[34] S. Chaudhuri, Y. Chen and E. Novak, Pair Correlation Function of Wilson Loops hep-th/9910183; S. Chaudhuri and E. Novak, Supersymmetric Pair Correlation Function of Wilson Loops hepth/0002046. 